\title{
The Role of Polymerase Chain Reaction (PCR) in Diagnosis of Spine Tuberculosis after Pre-operative Anti-tuberculosis Treatment
}

\author{
AH Rasit, MS Orth (UKM), SF Ibrahim*, MSc Biology, CC Wong**, MS Orth \\ Department Orthopaedics, Universiti Malaysia Sarawak, Kuching, Malaysia \\ *Department of Para-clinical Sciences, Universiti Malaysia Sarawak, Kuching, Malaysia \\ **Department Orthopaedics, Sarawak General Hospital, Kuching, Malaysia
}

\begin{abstract}
Objective: The aim of this study was to evaluate the role of polymerase chain reaction (PCR) in the diagnosis of spinal tuberculosis after 2 weeks of preoperative anti-tuberculosis treatment and to compare PCR to the Löwenstein - Jensen Culture (LJC) and histopathological examination (HPE) methods. Methods: Twenty-five patients were included in this study. Sixteen patients were diagnosed and treated for spinal tuberculosis based on clinical and radiological evidence. Nine patients were controls. The LJC method and HPE of the specimen were performed according to hospital protocol. PCR was performed using primer encoding insertion of sequences IS6110 for mycobacterium tuberculosis complex. Clinical findings and radiological features were the gold standard for comparison. Results: PCR results were 15 positive and one negative. The sensitivity and specificity of PCR was $94 \%$ and $100 \%$ respectively (with $95 \%$ confidence interval [CI] $67 \%$ to $99 \%$ and $63 \%$ to $100 \%$, respectively). HPE results showed 13 were positive and 3 negative in the spinal tuberculosis group; for the control group, all were negative. Sensitivity and specificity value of HPE was $82 \%$ and $100 \%$ respectively (with $95 \%$ confidence interval [CI] $54 \%$ to $95 \%$ and $63 \%$ to $100 \%$, respectively). Use of LJC showed only one was positive and 15 were negative in the spinal tuberculosis group whole all nine in the control group were negative. Sensitivity and specificity value of LJC was $6 \%$ and $100 \%$ respectively (with $95 \%$ confidence interval $[\mathrm{CI}] 0.3 \%$ to $32 \%$ and $63 \%$ to $100 \%$, respectively). Conclusion: Our findings showed that the PCR for Mycobacterium tuberculosis is reliable as a method for diagnosis of spinal tuberculosis, even after of 2 weeks of anti-TB treatment, with an overall sensitivity of $94 \%$ and specificity of $100 \%$.
\end{abstract}

Key Words:

Polymerase Chain Reaction, Tuberculosis, Spinal Tuberculosis

\section{INTRODUCTION}

Early treatment of spinal tuberculosis with anti-tuberculosis drugs is an important in disease management. In fact, early treatment is typically initiated diagnosis on clinical and radiological evidence without waiting for culture results, especially in areas endemic for $\mathrm{TB}$ and underdeveloped countries. The he gold standard of diagnosis by culture of the tuberculous organism is no longer practical currently as extended periods between 6 to 13 weeks are often needed to grow and identify the organism. Furthermore, the sensitivity of the culture $r$ can be as low as $50 \%^{1,2,3}$. Therefore, for purposes of this study the gold standard for diagnosis for spinal tuberculosis is based on clinical and radiological features.

Secondly, early treatment with anti-TB drugs is important for prevention of serious complications such as paraplegia; as reported in previous studies, the incidence of paraplegia ranges between $10 \%-44 \%$ of the spinal TB cases ${ }^{2,3}$. Lastly, if not treated early, this disease poses the risk of transmission to the medical personnel especially when handling the contaminated materials during surgical intervention ${ }^{4}$.

Recently, polymerase chain reaction (PCR) has been utilized for rapid identification of mycobacteria ${ }^{5,6,7}$. Studies of pulmonary and extrapulmonary tuberculosis have indicated that PCR is more specific and sensitive than other diagnostic methods. To date, there are no published studies that have assessed the role of PCR in the diagnosis of spinal tuberculosis after treated with anti-tuberculosis drugs has been initiated. This aim of this study was to evaluate the role of PCR in the diagnosis of spinal tuberculosis 2 weeks of treatment with anti-tuberculosis drugs is initiated, and then followed by radical surgical intervention.

\section{MATERIALS AND METHODS}

The criteria for diagnosis of spinal tuberculosis based on clinical and radiological findings are: at least 2 major +3 
Table I: Clinical and radiological features for the diagnosis of tuberculosis

\begin{tabular}{|l|l|}
\hline Clinical Features & Major: \\
& a. History of chronic back pain for more than 3 months \\
& b. Constitutional symptoms; low grade fever, night sweat, loss of appetite and weight loss \\
& c. Elevated erythrocyte sedimentation rate \\
Minor: & \\
& a. History of previous tuberculosis/contact with tuberculosis patient. \\
& b. Gibbous deformity \\
& c. Neurological deficit \\
& d. Cold abscess \\
e. Positive mantoux test. & a. Paradiscal lesion (loss of definition of the paradiscal margin of vertebral bodies). \\
Radiological Features & b. Loss of anterior vertebra \\
& c. Narrowing of the joint space \\
& d. Para vertebra shadow
\end{tabular}

Table II: Result of the PCR, Sensitivity $=94 \%$, Specificity $=100 \%$,

\begin{tabular}{|lccc|}
\hline Polymerase Chain Reaction & Spine Tuberculosis group & Control group & Total \\
\hline Positive & 15 & 0 & 15 \\
Negative & 1 & 9 & 10 \\
Total & 16 & 9 & 25 \\
\hline
\end{tabular}

Table III: Result of the HPE, Sensitivity $=82 \%$, Specificity $=100 \%$,

\begin{tabular}{|lccc|}
\hline Histopathological examination & Spine Tuberculosis group & Control group & Total \\
\hline Positive & 13 & 0 & 13 \\
Negative & 3 & 9 & 12 \\
Total & 16 & 9 & 25 \\
\hline
\end{tabular}

Table IV: Result of the LJC, Sensitivity $=6 \%$, Specificity $=100 \%$,

\begin{tabular}{|lccc|}
\hline Lowenstein-Jensen Culture & Spine Tuberculosis group & Control group & Total \\
\hline Positive & 1 & 0 & 1 \\
Negative & 15 & 9 & 24 \\
Total & 16 & 9 & 25 \\
\hline
\end{tabular}

minor clinical features and at least 2 radiological findings (Table I). The clinical findings and radiological features are the gold standard for the analysis of specificity and sensitivity of this study.

\section{Study Sample}

The study sample consisted of 25 patients. Inclusion criteria consisted of patients with a surgically treated spinal condition. Exclusion criteria consisted of patients treated only with conservatively interventions. Sixteen patients were diagnosed with spinal tuberculosis based on clinical and radiological (spinal tuberculosis group) findings; nine idiopathic scoliosis patients were included as the control group.

\section{Specimens}

All specimens were collected during surgery. Informed written consent was obtained from all study participants. The specimens were sent for PCR, Löwenstein - Jensen Culture
(LJC) and histopathological examination (HPE). All specimens were stored at $-20^{\circ} \mathrm{C}$ if not immediately processed in a sterile Eppendorf tube bottle without formalin.

\section{Löwenstein-Jensen Culture}

LJC was performed according to hospital protocol. Specimens were inoculated into two Löwenstein-Jensen medium culture bottles and incubated at $37^{\circ} \mathrm{C}$ for up to eight weeks. Solid medium slants cultures were considered positive when visible colonies grew. The colonies were further confirmed as mycobacteria using Ziehl-Neelsen stain.

\section{Polymerase Chain Reaction}

DNA extraction (with modification) as performed using Genispin Bacterial DNA kit (BST Techlab, cat no: DX1506$5 \mathrm{~N}, 50$ preps). Oligonucleotide primers for Mycobacterium tuberculosis (described by Eisenach et al) were used for amplification ${ }^{8}$. This primer is designed to detect the 
repetitive sequence of IS6110, and can amplify the 123bp region of IS6110 specific for Mycobacterium tuberculosis complex (Mycobacterium tuberculosis, Mycobacterium bovis, Mycobacterium bovis, Mycobacterium africanum and Mycobacterium microti). The primer sequences are 5'CCTGCGAGCGTAGGCGTCGG-3' and 5'CTCGTCCAGCGCCGCTTCGG-3'.

Amplification steps included use of the Mtb68 program on a MJ Research thermocycler (PTC 200) in a general research lab, located in the medical schoo. Initial denaturation at $94^{\circ} \mathrm{C}$ was performed for 10 minutes followed by denaturation at $94^{\circ} \mathrm{C}$ for 1 minute, annealing at $68^{\circ} \mathrm{C}$ for 1 minute in 39 cycle, extension at $72^{\circ} \mathrm{C}$ for 1 minute, and finally extension at $72^{\circ} \mathrm{C}$ for 10 minutes. This amplification takes a total of 3 hours. The PCR product samples are then run on $2.2 \%$ agarose gel in $0.5 \mathrm{X}$ TBE buffer (using 10X TBE buffer (BST Techlab, Cat. No. BST-1201L) and 2.2g agarose powder (Promega, Cat. No. V3125)). The PCR products, positive and negative controls, and 100bp DNA ladder (MBI Fermentas, \#SM0243, and $0.1 \mathrm{mg} / \mathrm{ml}$ ) were electrophoresed at $110 \mathrm{~V}$ for approximately 10 minutes. The gels were visualized under ultraviolet light.

\section{Treatments}

All spinal tuberculosis patients were given at least 2 weeks of anti-tuberculosis drugs followed by radical surgical procedure. Criteria for the radical surgical procedure were progressive neurological deficit, large paravertebral abscess or severe kyphosis. All spinal TB patients required radical surgery, radical anterior decompression and strut grafting. Patients were followed up with erythrocyte sedimentation rate (ESR) and spinal $x$-ray postoperatively.

If the result of LJC, HPE or PCR was positive for mycobacterium tuberculosis, the anti-tuberculosis drugs were continued for 12 months. Treatment was also continued if the patient showed improvement of the condition after 2 months, even if any of the methods showed a negative result. If another diagnosis resulted from any of the three methods and other results were negative for mycobacterium tuberculosis with no improvement after 2 months of treatment, anti-tuberculosis drugs were discontinued immediately.

\section{RESULTS}

There was an average of a 21 month history of backache in the control group compared to an average of four months in the spinal tuberculosis group. Thirty-five per cent and $17 \%$ of the spinal TB group had a history of contact with a TB patient and pulmonary TB patient respectively. The incidence of gibbous deformity, paravertebral abscess; neurological deficit and constitutional symptoms in the spinal tuberculosis group were $47 \%, 59 \%, 17 \%$ and $41 \%$ respectively. The average ESR in the spinal tuberculosis group was $67 \mathrm{~mm} /$ hour whereas it averaged $36 \mathrm{~mm} /$ hour in the control group.

The results of the PCR for mycobacterium tuberculosis showed 15 were positive and one was negative in the spinal tuberculosis group, while 9 of 9 were negative in the control group. The diagnostic sensitivity and specificity of PCR was $94 \%$ and $100 \%$ respectively (Table II).

For HPE, in the spinal tuberculosis group, thirteen were positive with HPE of spinal tuberculosis and three were negative; all of the patients in the control groups were negative for tuberculosis using the HPE method. Diagnostic sensitivity and specificity of HPE was $82 \%$ and $100 \%$ respectively (Table III). One out of sixteen specimens was positive for culture in the spinal tuberculosis group, and the entire control group gives a negative result after 2 months of incubation. The diagnostic sensitivity of LJC was $6 \%$ and $100 \%$ in clinically diagnosed and treated spinal tuberculosis, respectively (Table IV).

All patients in the spinal tuberculosis underwent radical surgery using an anterior approach and iliac bone graft; all patients in the control group underwent surgery using a posterior approach. Five patients in the spinal tuberculosis group, required supplementary instrumentation using pedicle screw fixation.

All patients were prescribed 12 months of anti-tuberculosis drug therapy using any of regimens recommended by the Malaysian Ministry of Health.

\section{DISCUSSION}

The clinical and radiological evidence remains the fundamental deciding factors for early management of spinal tuberculosis in endemic areas such as Malaysia because of the low sensitivity and extended timeframe needed to grow and identify M. tuberculosis. In the present series of 16 patients with clinical and radiologically diagnosed and treated spinal tuberculosis, only one patient had a positive culture after 4 weeks of incubation. The low sensitivity of culture in this series may be due to the character of the organism itself; furthermore the specimen was taken after 2 weeks of anti-tuberculosis treatment.

The sensitivity of PCR is ranged between $42 \%$ and $93 \%$ in culture positive specimens $2,9,10,11,12,13$. PCR results can be ready within three days, enabling earlier diagnosis and treatment ${ }^{14,15,16}$. This is similar to other reported results using IS6110 as a target site for laboratory diagnosis ${ }^{16}$. Positive PCR may indicate the presence of non-viable acid-fast bacilli $(A F B)$, particularly in patients with a history of TB or anti-tuberculosis drugs given prior to specimen collection. It is also not possible to differentiate live from dead organisms or judge disease activity. To overcome this issue, clinical 
judgment and other laboratory tools such as ESR and spinal $\mathrm{x}$-ray must be used to monitor the activity of infection and response to the treatment.

For the current study, protocol called for early treatment with preoperative anti-tuberculosis drugs for at least 2 weeks prior to surgery - this approach has several advantages. First, stopping progression of the disease is most important, and second it is of utmost importance to prevent major complications such as paraplegia and severe deformity. Further, specimens are more easily collected during open surgery as compared to needle biopsy. Despite the 2 weeks of anti-tuberculosis treatment, PCR and HPE result were sensitive and specific tools for diagnosis of spinal tuberculosis as compared to LJC. Lastly, 2 weeks of preoperative anti-tuberculosis treatment reduced anxiety and fear of the staff regarding risk of disease transmission especially when handling the infected material during the operative procedure.

The high sensitivity and specificity of PCR in our study may be due in part to adequate specimen collection during surgical intervention especially as compared to conventional needle biopsy technique. The high sensitivity (94\%) and specificity $(100 \%)$ are similar to results reported in other studies. Ichiyama et al reported $97 \%$ sensitivity and $98.7 \%$ specificity, however Vuorinen et al reported slightly lower sensitivity of $84.6 \%$ and specificity of $99.1 \%$ using similar commercially available PCR (Amplicor) with IS6110 as a target site for laboratory diagnosis ${ }^{17,18}$.
Vivek et al reported sensitivity of PCR using spinal samples was $90 \%$ and had a low rate of false positives $(13.63 \%)^{19}$. They concluded that PCR is an effective method for diagnosis tuberculosis and anti-tuberculosis treatment can be started if PCR is positive. However, Kiran et al 20 reported $74.1 \%$ sensitivity (with 95\% Confidence Interval [CI] 53.4; 88.1 ) and $96.1 \%$ specificity (with $95 \%$ CI, 88.2; 98.9) using PCR. They concluded that although HPE is a cost- effective method, early PCR results enables clinicians to start treatment in advance of differential diagnosis to prevent irreversible complications.

We concur with Vivek and Kiran study that early antituberculosis treatment is essential for current clinical management. Our study showed that even after 2 weeks of anti-tuberculosis drugs treatment before surgical intervention and sample collection, PCR analysis resulted in high sensitivity. This evidence supports HPE results.

\section{CONCLUSION}

Polymerase chain reaction analysis is a reliable method for for Mycobacterium tuberculosis diagnosis of spinal tuberculosis even after 2 weeks of anti-tuberculosis treatment, with an overall sensitivity of $94 \%$ and specificity of $100 \%$.

\section{ACKNOWLEDGEMENT}

We acknowledge the financial support rendered by Unimas through the Fundamental Research Grant No. 01(15)/290/2002(29). 


\section{REFERENCES}

1. Daniel TM. The rapid diagnosis of tuberculosis. A selective review. J Lab Clin Med 1990; 116(3): 277-82.

2. Jain AK, Jena SK, Singh MP, Dhammi IK, Ramachadran VG, Dev G. Evaluation of clinico-radiological, bacteriological, serological, molecular and histological diagnosis.

3. Rasit AH, Razak M, Ting FSS. The pattern of Spinal Tuberculosis in Sarawak.

4. Gopinath KG, Siddique S, Kirubakaran H, Shanmugam A, Mathai E, Chandy GM. Tuberculosis among healthcare workers in a tertiary-care hospital in South India. J Hosp Infect 2004; 57(4): 339-42.

5. Shah S, Miller A, Mastellone A, Kim K, Colaninno P, Hochstein L et al. Rapid diagnosis of tuberculosis in various biopsy and body fluid specimens by the AMPLICOR Mycobacterium tuberculosis polymerase chain reaction test. Chest 1998; 113(5): 1190-4.

6. Fauville-Dufaux M, Vanfleteren B, De Wit L, Vincke JP, Van Vooren JP, Yates MD et al. Rapid detection of tuberculous and nontuberculous mycobacteria by polymerase chain reaction amplification of a 162 bp DNA fragment from antigen 85 . Eur J Clin Microbiol Infect Dis 1992; 11(9): 797-803.

7. Magana AD, Perera J, Gamage S, Chandrasekharan V. Low cost in house PCR for the routine diagnosis of extre-pulmonary tuberculosis. Inter J Tuberc Lung Dis 2008; 12(3): 275-80.

8. Eisenach KD, Cave MD, Bates JH, Crawford JT. Polymerase chain reaction amplification of a repetitive DNA sequence specific for Mycobacterium tuberculosis. J Infect Dis 1990; 161(5): 977-81.

9. Chan CM, Yuen KY, Chan KS, Yam WC, Yim KH, Ng WF et al. Single-tube nested PCR in the diagnosis of tuberculosis. $J$ Clin Pathol 1996; 49: 290-4.

10. Kearns AM, Freeman R, Steward M, Magee JG. A rapid polymerase chain reaction technique for detecting Mycobacterium tuberculosis in a variety of clinical specimens. J Clin Pathol 1998; 51: 922-4.

11. Kolk AH, Kox LF, van Leeuwen J, Kuijper S, Jansen HM. Clinical utility of the polymerase chain reaction in the diagnosis of extrapulmonary tuberculosis. Eur Respir J 1998; 11: 1222-6.

12. Bemer-Melchior P, Germaud P, Drugeon HB. Diagnosis of extrapulmonary tuberculosis by a commercial polymerase chain reaction kit. Pathol Biol (Paris) 1998; 46: 597-603.

13. Tortoli E, Tronci M, Tosi CP, Galli C, Lavinia F, Natili S et al. Multicenter evaluation of two commercial amplification kits (Amplicor, Roche and LCx, Abbott) for direct detection of Mycobacterium tuberculosis in pulmonary and extrapulmonary specimens. Diagn Microbiol Infect Dis 1999; 33: 173-9.

14. Pandey J, Talib VH. Laboratory diagnosis of tuberculosis: Use of ELISA and PCR. Indian J Pathol Microbiol 1993; 36: 512-8.

15. Jatana SK, Nair MN, Lahiri KK, Sarin NP. Polymerase chain reaction in the diagnosis of tuberculosis. Indian Pediatr 2000; 37: 375-82.

16. Almeda J, García A, González J, Quintó L, Ventura PJ, Vidal R et al. Clinical evaluation of an in-house IS6110 polymerase chain reaction for diagnosis of tuberculosis. Eur J Clin Microbiol Infect Dis 2000; 19: 859-67.

17. Ichiyama S, Iinuma Y, Tawada Y, Yamori S, Hasegawa Y, Simokata H et al. Evaluation of GenProbe amplified Mycobacterium tyberculosis direct test and Roche-PCR microwell plate hybridization method (Amplicor Mycobacterium) for direct detection of mycobacteria. J Clin Microbiol 1996; 34: 130.

18. Vourinen P, Miettinen A, Vuento R, Hallstrom O. Direct detection of Mycobacterium tuberculosis complex in respiratory specimens by Gen-Probe Amplified Mycobacterium Tuberculosis Direct Test and Roche Amplicor Mycobacterium Tuberculosis Test. J Clin Microbiol 1995; 33: 1856.

19. Pandey V, Chawla K, Acharya K, Rao S, Rao S. The role of polymerase chain reaction in the management of osteoarticular tuberculosis. Int Orthop 2009; 33: 801-5.

20. Chawla K, Gupta S, Mukhopadhyay C, Rao PS, Bhat SS. PCR for M. tuberculosis in tissue samples. J Infect Dev Ctries 2009; 3(2): $83-7$. 